\title{
Spinal epidural abscess in COVID-19 patients
}

\section{G. Talamonti ${ }^{1}$ [D $\cdot$ Davide Colistra $^{1} \cdot$ Francesco Crisà $^{1,3} \cdot$ Marco Cenzato $^{1} \cdot$ Pietro Giorgi $^{2} \cdot$ Giuseppe D'Aliberti $^{1}$}

Received: 21 June 2020 / Revised: 29 August 2020 / Accepted: 2 September 2020 / Published online: 10 September 2020

(c) Springer-Verlag GmbH Germany, part of Springer Nature 2020

\begin{abstract}
Objective To report the peculiarity of spinal epidural abscess in COVID-19 patients, as we have observed an unusually high number of these patients following the outbreak of SARS-Corona Virus-2.

Methods We reviewed the clinical documentation of six consecutive COVID-19 patients with primary spinal epidural abscess that we surgically managed over a 2-month period. These cases were analyzed for what concerns both the viral infection and the spinal abscess.

Results The abscesses were primary in all cases indicating that no evident infective source was found. A primary abscess represents the rarest form of spinal epidural abscess, which is usually secondary to invasive procedures or spread from adjacent infective sites, such as spondylodiscitis, generally occurring in patients with diabetes, obesity, cancer, or other chronic diseases.

In all cases, there was mild lymphopenia but the spinal abscess occurred regardless of the severity of the viral disease, immunologic state, or presence of bacteremia. Obesity was the only risk factor and was reported in two patients. All patients but one were hypertensive. The preferred localizations were cervical and thoracic, whereas classic abscess generally occur at the lumbar level. No patient had a history of pyogenic infection, even though previous asymptomatic bacterial contaminations were reported in three cases.

Conclusion We wonder about the concentration of this uncommon disease in such a short period. To our knowledge, cases of spinal epidural abscess in COVID-19 patients have not been reported to date. We hypothesize that, in our patients, the spinal infection could have depended on the coexistence of an initially asymptomatic bacterial contamination. The well-known COVID-19-related endotheliitis might have created the conditions for retrograde bacterial invasion to the correspondent spinal epidural space.

Furthermore, spinal epidural abscess carries a significantly high morbidity and mortality. It is difficult to diagnose, especially in compromised COVID-19 patients but should be kept in mind as early diagnosis and treatment are crucial.
\end{abstract}

Keywords COVID-19 $\cdot$ SARS-CoV-2 $\cdot$ Spinal abscess $\cdot$ Spinal epidural abscess $\cdot$ Spinal infection

\section{Introduction}

The first case of Coronavirus Disease 2019 (COVID-19) was diagnosed in Lombardy on February 21, 2020. Afterwards, the contagion rapidly spread throughout the region. Up to today (June 12, 2020), 90,932 Lombards have contracted COVID-19 and 16,374 have died, which makes Lombardy

G. Talamonti

tala_nch@yahoo.it

1 Departments of Neurosurgery, ASST Niguarda, Piazza Ospedale Maggiore 3, 20162 Milan, Italy

2 Department of Orthopedics, ASST Niguarda, Milan, Italy

3 Università Di Milano, Milan, Italy (a region of about 10 million inhabitants) one of the most plagued areas of the world. The Lombard Health System had to be reorganized to face the outbreak: our department suspended all elective procedures and managed only neurosurgical emergencies. During the last three months, six patients with SARS-Corona Virus-2 (SARS-COV-2) were referred to us for acute spinal cord syndrome due to primary spinal epidural abscess (SEA) [1]. No adjacent spondylodiscitis or other infection sources were evident. This time-concentrated incidence of primary SEA is quite unusual in our practice, and a relationship with COVID-19 is conceivable. To our knowledge, to date there are no published cases of SEA in patients with COVID-19. 


\section{Materials and methods}

During the peak of the epidemic (March to May 2020), we had to surgically manage six COVID-19 patients with SEA. Their clinical charts, radiological documentation, and surgical reports were carefully reviewed looking for possible peculiarities for what concerns both the viral infection and the neurological disease. The main clinical features are summarized in Table 1.

Four men and two women (mean age $=58.5$ years) were referred to us because of progressively worsening spinal cord dysfunction. All these patients presented with few day histories of back pain and a diagnosis of COVID-19.

\section{Viral infection}

On admission to our department, the diagnosis of COVID19 had already been ascertained in three patients and was highly suspected in a fourth.

In particular, one patient was still hospitalized because of interstitial pneumonia (IP), whereas two patients had recently been discharged after recovery from IP. These three individuals had had positive nasopharyngeal swabs for SARS-COV-2 and their chest-computed tomography (CT) had shown the typical ground-glass appearance. Therapy had included tocilizumab, antibiotics, corticosteroids, anticoagulants, and antiviral agents. The fourth patient had been admitted to another department because of dyspnea, fever, and anosmia. He had a known history of deep vein thrombosis and chest-CT had shown pulmonary infarction and IP. Nasopharyngeal swabs were negative. Serologic tests were initially unavailable. Subsequently, such tests were planned but never performed because this patient deteriorated to a pre-agonic state owing to new pulmonary infarction. These four patients had required respiratory assistance (CPAP, NIMV, or intubation), but two had completely overcome the disease. The remaining two patients had histories of recent flu episodes but never presented clear symptoms of COVID19. They had negative nasopharyngeal swabs, but serologic tests revealed exposure to SARS-COV-2.

\section{Spinal epidural abscess}

On admission to our department, three patients were quadriparetic, two were paraparetic, and one was paraplegic. Neurological conditions were rapidly worsening in all cases. All patients underwent magnetic resonance imaging (MRI) (Fig. 1), which showed epidural abscesses at cervical (3 patients), thoracic (2 patients), and lumbar (1 patient) levels. In no case did the MRI show spondylodiscitis or any other possible sources of infection. Mild fever was reported only in one patient. Slight neutrophil increase and lymphopenia were present in all cases. Procalcitonin was increased in three cases and C-reactive Protein (CRP) was increased in all cases but one.

All patients underwent emergency hemi-laminectomy with pus drainage and spinal cord decompression.

\section{Results}

In all cases, cultural assay of the surgical material revealed methicillin-sensitive Staphylococcus aureus (MSSA). During the following days, MSSA was also found in the blood of two patients despite no sign of sepsis being evident. In all cases, antibiogram allowed adequate and effective antibiotic therapy. Postoperatively, the infectious disease specialist carefully searched for possible hidden sources of infection, but clinical examination, total body CT-scan, orthopantomography, and cultural assays of several fluids and secretions were all negative. No other associated infections were found. The previous clinical histories were also carefully examined. All six patients had never presented clinical evidence of pyogenic infection. However, when we managed them for the SEA, we retrospectively reconstructed that, some weeks before, Staphylococcus aureus (SA) had been found in the expectoration and oropharyngeal swabs of two and one patients, respectively. At that time, the patients were asymptomatic for bacterial infection and these findings were interpreted as sample contamination. No patient was an intravenous drug abuser. Predisposing factors for SEA (obesity and obesity plus diabetes) were reported in two cases only. Chronic arterial hypertension was present in five cases.

Repeated MRI documented the abscess resolution in all cases. Within a couple of weeks all patients were referred to rehabilitation units. One month later, the patient with known deep vein thrombosis was still quadriplegic, suffered a new pulmonary infarction, and rapidly deteriorated. Two months after surgery, partial recovery was evident in four patients, whereas one was completely paraplegic. Furthermore, no patient completely recovered from the initial spinal cord syndrome.

\section{Discussion}

\section{SEA}

Risk factors for SEA include diabetes, immunosuppressed state, intravenous drug abuse, chronic renal or liver failure, spinal surgery, obesity, and bacteremia [2-9]. The incidence of SEA is quite low ranging from 1.2 to 3 per 10,000 hospitalized patients $[3,7,9]$. Cases of primary SEA, which are 


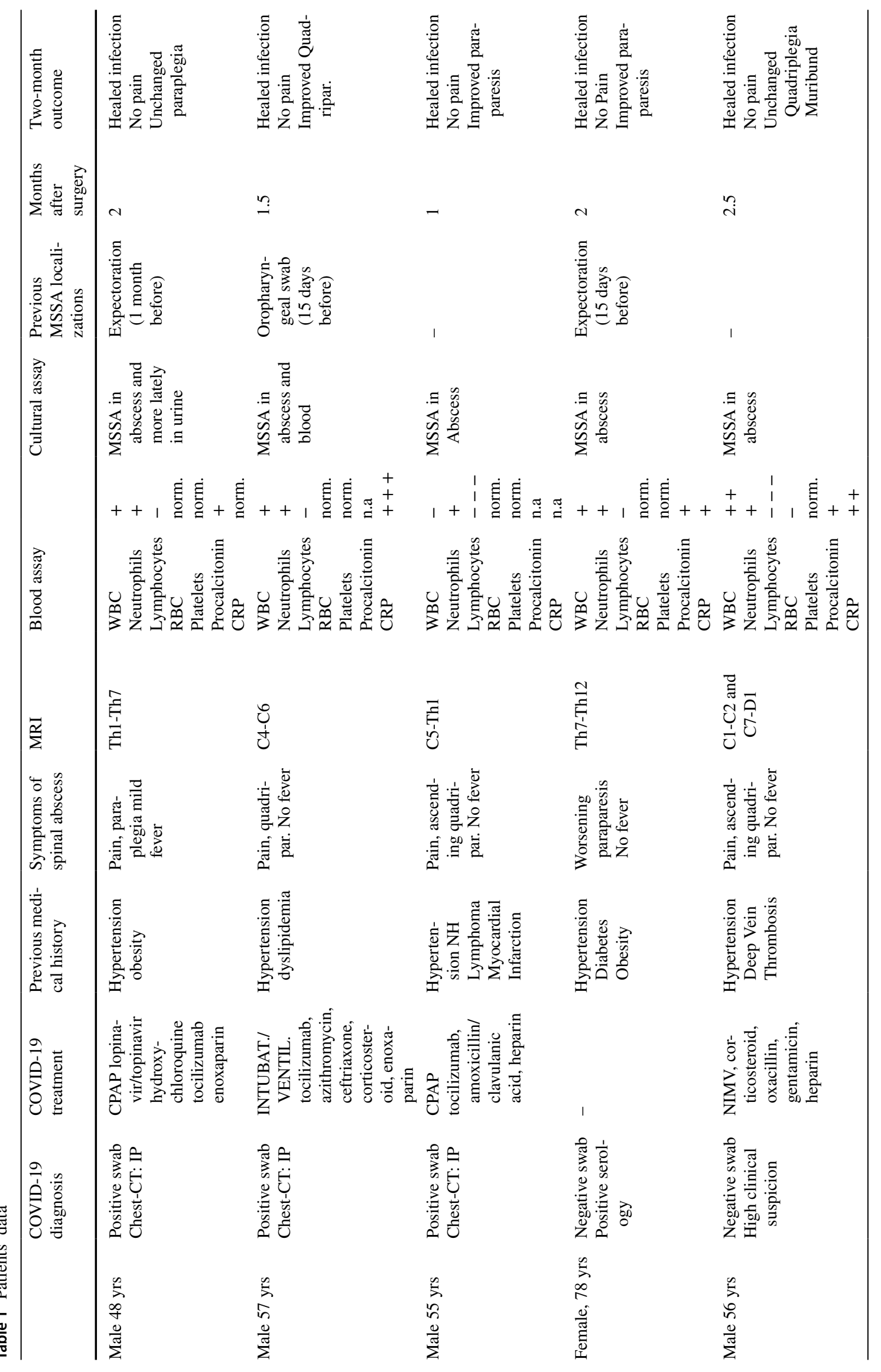


cases without any evident infective source, are even rarer accounting for $20 \%$ of all SEAs [1,2].

SA, usually the MSSA, is responsible for $70 \%$ of cases $[3,4,6,7,9]$.

Pain is constant, fever is present in less than $50 \%$ of cases, and true neurological deficits affect a minority of patients $[4-6,9,10]$. Onset of symptoms may be sudden, or slowly progressive but back pain often evolves to paraplegia within a few days [7]. Accordingly, early diagnosis is crucial, but it is difficult, and half of cases are initially misdiagnosed $[3-7,9]$.

The white blood cell (WBC) count is normal in about half of patients, while erythrocyte sedimentation rate and CRP are generally elevated $[4,5,7,9-11]$. Bacteremia causing or arising from SEA is detected in $60 \%$ of patients [5-7].

MRI with contrast is the diagnostic method of choice: SEA is generally seen as a $\mathrm{T} 1$ hypointense, $\mathrm{T} 2$ hyperintense mass with an enhancing capsule in the epidural space [4-7]. Most SEAs are lumbar and posterior because infections are more likely in larger fatty epidural spaces [5].

Decompressive laminectomy and drainage together with systemic antibiotics are mandatory in patients with neurological symptoms [5, 9]. Pending the identification of the causative organism, empiric therapy should start using broad spectrum antibiotics [5]. There are no guidelines for the duration of therapy, but patients typically require $4-8$ weeks of therapy [3-7, 9]

Postoperative recovery depends on age, health status, comorbidities, and history duration [12-15] but above all on the patient's neurologic status immediately before surgery $[2,4,5,7]$. Despite recent improvements, outcomes of SEA remain poor, with mortality ranging from 5 to $23 \%$ and neurological morbidity ranging from 4 to $55 \%[4-7,9,16]$.

\section{COVID-19}

COVID-19 is primarily a respiratory tract infection with significant impaction on different systems [17-19]. Recent studies show that the SARS-COV-2 provokes diffuse damage to the vascular endothelium triggering a sort of disseminated intravascular coagulation [19-21]. In a large autoptic series [21], all cases presented a more or less degree of endothelial damage and arteriolar thrombosis was evident in $87 \%$ of cases. The IP that complicates COVID-19 can be de facto considered as diffuse micro-infarctions of the lung. Lymphocytes and monocytes often decrease with a possible impaired immune response to exogenous infective agents [17, 22, 23]. Bacterial infections have been reported in half of patients [18-20, 22, 23]. From a practical point of view, COVID-19 may consist of a complex clinical situation including disseminated micro-embolisms, bleeding diathesis, diffuse vasculitis, and autoimmune aggression with 
Fig. 1 a Chest CT-scan obtained in a 48-year-old man to control the evolution of Interstitial Pneumonia due to COVID19. The "atoll sign" (arrow), that is expression of organizing pneumonia, is evident. b Spinal MRI obtained the following day showing an epidural abscess extending from Th1 to Th7 (arrows). c Postoperative MRI showing the good drainage of the abscess with three-level emi-laminectomy
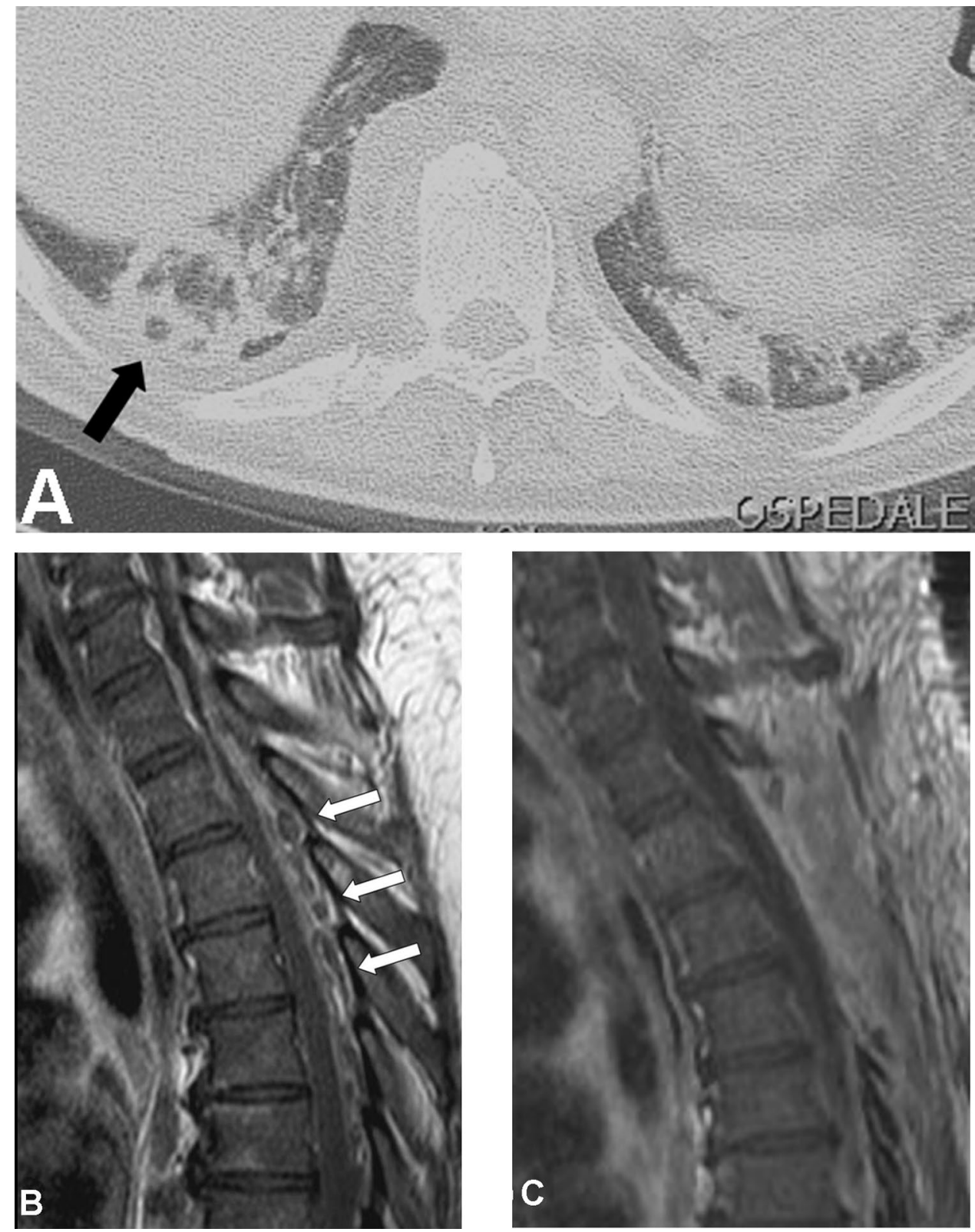

decreased antibacterial defense. However, asymptomatic or paucisymptomatic cases are common.

\section{COVID-19 and primary SEA}

During the last ten years, we surgically treated a total of seven patients with primary SEA that means without spondylodiscitis or an evident infective source. Following the outbreak of COVID-19, we received six patients in a couple of months. Three of these patients complained of fullblown severe COVID-19. In another patient, the diagnosis was only based on clinical symptoms, but we think it was highly probable. In two patients, the viral infection was almost asymptomatic and was just revealed by the serologic tests. Accordingly, the clinical severity of COVID-19 was not correlated to the SEA occurrence. Moreover, when SEA occurred, only two patients were still fighting against active COVID-19.

These six patients presented some differences from classical SEA patients: they were relatively younger; none was a drug abuser; only two were obese and only one of these was diabetic; the typical lumbosacral location was present in only one case. However, apart from the relatively unusual cervical and thoracic locations, there was no peculiar MRI feature in comparison with classical SEAs. All these patients had lymphopenia and three had previously received immunomodulators to counter the viral infection. Mild immunodeficiency cannot be excluded even in the two patients 
who were asymptomatic for COVID-19. Nonetheless, we do not believe that immunodeficiency played a major role in the SEA development. As previously mentioned, an immunocompromised state represents a risk factor for developing SEA and lymphopenia is quite common in COVID-19 patients. Accordingly, an increased SEA incidence could be expected in COVID-19 patients. Conversely, to our knowledge, cases of SEA in COVID-19 patients have not yet been published. Theoretically, SEA could have been underdiagnosed in comatose or severely compromised COVID-19 patients. Otherwise, SEA simply may not have been reported because physicians focused on other aspects of the disease.

In two patients, MSSA was also subsequently found in blood cultures. Perhaps, bacteremia could have caused the SEA, but it is also possible that the bacterium secondarily entered the blood from the SEA [5-7]. Both these patients were apyretic, never presented signs of sepsis, and WBCs and neutrophils were just moderately increased.

None of the patients had ever presented clinical evidence of pyogenic infection. Nosocomial infections may be perhaps suspected in the two hospitalized patients, but these presented no sign of sepsis or other infection. Three patients had a history of recent asymptomatic SA presence in the pharynx and expectoration, which had been interpreted as sample contamination. When SEA occurred, neither pharyngeal nor pulmonary infections were evident. Interestingly, within a few weeks, the two patients with contaminated expectoration developed thoracic SEA, the one with a contaminated swab suffered from cervical SEA. Taking into account that cervical and thoracic locations are relatively unusual for SEA, retrograde spinal invasion is conceivable.

As previously mentioned, the coronavirus is typically responsible for diffuse endothelial damage [20, 21]. In two of our patients with IP, the chest-CT-scan (Fig. 1) also showed the "atoll-sign", which is a well-known expression of inflammation and granulomatous reaction in organizing pneumonia and is classically associated to angio-invasive agents [24]. All patients but one had a history of arterial hypertension. We wonder if arterial hypertension could have played a role in damaging the vascular endothelium, thus favoring the vascular penetration of SA even in the absence of a clear SA infection. In this way, SA could have retrogradely reached the correspondent spinal epidural space causing progressive cellulitis of the epidural fat with the ultimate formation of the SEA.

If this was the case, the higher than normal SEA incidence in this population might be explained. Of course, we are not stating that COVID-19 was responsible for SEA development but a role can be hypothesized. The viral infection might create the conditions for spinal invasion in subjects that are predisposed owing to the presence of a bacterium in a given location. This might also account for the cervical and thoracic SEAs that are relatively uncommon in classic SEA patients. Even the late onset of SEA following recovery from COVID-19 might be explained by the time to retrogradely invade the epidural space.

COVID-19 patients may present problems that can seriously hamper surgeries [25]. However, we did not encounter particular surgical problems in these six patients, whose platelets were normal and immunological, respiratory, and circulatory states were acceptable. Despite relatively prompt treatment, no patient completely recovered from the spinal cord damage. Since the preoperative status is the main determinant of a favorable outcome $[2,4,5,7]$ and SEA may be encountered at unexpected rates, careful neurological examination of COVID-19 patients is mandatory.

\section{Conclusion}

Following the outbreak of COVID-19, we noticed an unusual high incidence of primary SEA which indeed should be quite uncommon in non-drug abuser patients. In our mind, if the SEA development were just related to an immunocompromised state or to simple nosocomial superinfections, we should encounter much more patients with SEA considering the magnitude of the pandemic. Instead, no case has yet been published even though we suspect that the incidence might be higher.

We hypothesize that SEA may develop because an asymptomatic bacterial colonization co-exists with damage to the vascular endothelium induced by COVID-19 at the same level. This could favor retrograde spinal invasion at the correspondent level.

Since the outcome of SEA often remains poor mainly because of delayed diagnosis and treatment, physicians should be aware that COVID-19 patients may have some greater risk of SEA than the general population.

Acknowledgements The Authors thank the other components of the Spine Team of the ASST Niguarda of Milan: Dr Giuseppe Schirò and Dr Enrico Gallazzi from the Orthopedic Department, Dr Fabio Giuseppe Villa from the Neurosurgical Department.

Funding The authors declare that they received no funding.

Data availability All the data supporting our findings are contained within manuscript.

Code availability Not applicable.

\section{Compliance with ethical standards}

Conflicts of interest The authors declare no conflicts of interest associated with this study.

Ethical approval This study was approved by the ASST Niguarda Ethics Committee 
Informed consent This is a retrospective work that does not require informed consent.

Consent for publication All authors have approved publication of the manuscript.

\section{References}

1. Khan SH, Hussain MS, Griebel RW et al (2003) Title comparison of primary and secondary spinal epidural abscesses: a retrospective analysis of 29 cases. Surg Neurol 59:28-33. https://doi. org/10.1016/s0090-3019(02)00925-4

2. Wang Z, Lenehan B, Itshayek E et al (2012) Primary pyogenic infection of the spine in intravenous drug users. Spine 37:685692. https://doi.org/10.1097/BRS.0b013e31823b01b8

3. Ameer MA, Knorr TL, Mesfin FB (2020) Spinal epidural abscess. StatPearls. Treasure Island (FL): StatPearls Publishing

4. Chow F (2018) Brain and spinal epidural abscess. Continuum (Minneap Minn) 24:1327-1348. https://doi.org/10.1212/ CON.0000000000000649

5. Darouiche RO (2006) Spinal epidural abscess. N Engl J Med 355:2012-2020. https://doi.org/10.1056/NEJMra055111

6. DeFroda SF, DePasse JM, Eltorai AEM et al (2016) Evaluation and management of spinal epidural abscess. J Hosp Med 11:130 135. https://doi.org/10.1002/jhm.2506

7. Johnson KG (2013) Spinal epidural abscess. Crit Care Nurs Clin North Am 25:389-397. https://doi.org/10.1016/j.ccell 2013.04.002

8. Schoenfeld AJ, Wahlquist TC (2015) Mortality, complication risk, and total charges after the treatment of epidural abscess. Spine J 15:249-255. https://doi.org/10.1016/j.spinee.2014.09.003

9. Vakili M, Crum-Cianflone NF (2017) Spinal epidural abscess: a series of 101 cases. Am J Med 130:1458-1463. https://doi. org/10.1016/j.amjmed.2017.07.017

10. Reihsaus E, Waldbaur H, Seeling W (2000) Spinal epidural abscess; a meta-analysis of 915 patients. Neurosurg Rev 232:175204. https://doi.org/10.1007/p100011954

11. Kolinsky DC, Liang SY (2018) Musculoskeletal infections in the emergency department. Emerg Med Clin North Am 36:751-766. https://doi.org/10.1016/j.emc.2018.06.006

12. D'Aliberti G, Talamonti G, Villa F et al (2012) The anterior stand-alone approach (ASAA) during the acute phase of spondylodiscitis: results in 40 consecutively treated patients. Eur Spine J 21:75-82. https://doi.org/10.1007/s00586-012-2238-7
13. Berwick BW, Luo TD, Sun KW et al (2019) Epidural abscess in the lumbar spine: a single institution's experience with nonsurgical and surgical management. J Surg Orthop Adv 28:224-231 (PMID: 31675300)

14. Khursheed N, Dar S, Ramzan A et al (2017) Spinal epidural abscess: report on 27 cases. Surg Neurol Int 8:240. https://doi. org/10.4103/sni.sni_105_17

15. Suppiah S, Meng Y, Fehlings MG et al (2016) How best to manage the spinal epidural abscess? A current systematic review. World Neurosurg 93:20-28. https://doi.org/10.1016/j.wneu.2016.05.074

16. Kang T, Park SY, Lee SH et al (2019) Spinal epidural abscess successfully treated with biportal endoscopic spinal surgery. Medicine (Baltimore) 98:e18231. https://doi.org/10.1097/MD.00000 00000018231

17. Mehta P, McAuley DF, Brown M et al (2020) COVID-19: consider cytokine storm syndromes and immunosuppression. Lancet 395:1033-1034. https://doi.org/10.1016/S0140-6736(20)30628-0

18. Terpos E, Ntanasis-Stathopoulos I, Elalamy I et al (2020) Hematological findings and complications of COVID-19. Am J Hematol. https://doi.org/10.1002/ajh.25829

19. Zhang Y, Geng X, Tan Y et al (2020) New understanding of the damage of SARS-CoV-2 infection outside the respiratory system. Biomed Pharmacother. https://doi.org/10.1016/j.bioph a.2020.110195

20. Lodigiani C, Iapichino G, Carenzo L et al (2020) Venous and arterial thromboembolic complications in COVID-19 patients admitted to an academic hospital in Milan, Italy. Thromb Res 191:9-14. https://doi.org/10.1016/j.thromres.2020.04.024

21. Carsana L, Sonzogni A, Nasr A et al (2020) Pulmonary postmortem findings in a series of COVID-19 cases from northern Italy: a two-centre descriptive study. Lancet Infect Dis. https:// doi.org/10.1016/S1473-3099(20)30434-5

22. Wang L, He W, Yu X et al (2020) Coronavirus disease 2019 in elderly patients: Characteristics and prognostic factors based on 4-week follow-up. J Infect. https://doi.org/10.1016/j. jinf.2020.03.019

23. Yang X, Yu Y, Xu J et al (2020) Clinical course and outcomes of critically ill patients with SARSCoV2 pneumonia in Wuhan, China: a single centered, retrospective, observational study. Lancet Respir Med. https://doi.org/10.1016/S22132600(20)300795

24. Godoy MC, Viswanathan C, Marchiori E et al (2012) The reversed halo sign: update and differential diagnosis. Br J Radiol 85:12261235. https://doi.org/10.1259/bjr/54532316

25. Panciani PP, Saraceno G, Zanin L et al (2020) Letter: COVID-19 infection affects surgical outcome of chronic subdural hematoma. Neurosurgery. https://doi.org/10.1093/neuros/nyaa140 